\title{
Nutrient stress revamps cancer cell metabolism
}

\author{
Cell Research (2015) 25:537-538. doi:10.1038/cr.2015.38; published online 31 March 2015
}

Efforts to identify new therapeutic targets in cancer primarily focused on oncogenes and tumor suppressor genes, and their mechanisms of action. However, there is an emerging alternative strategy that involves identification of target proteins that are not encoded by oncogenes, but are, nonetheless, required to accommodate cancer-specific stresses.

One of the most interesting, and possibly richest, sources for new therapeutic targets is the cellular machinery regulating cancer cell metabolism. Tumor cells normally rely on aerobic glycolysis to maintain cell growth and proliferation, even in the presence of normal concentrations of oxygen [1]. This paradoxical situation makes cancer cells relatively resistant to growth inhibition in conditions of oxygen deprivation, and provides a way to rapidly produce the cellular energy and metabolites required for the high rate of anabolism that drives the dramatically increased proliferation of cancer cells [1]. This is an efficient metabolic mechanism as long as cancer cells have access to a constant supply of glucose. However, cancer cell addiction to a high glucose supply makes them vulnerable and, therefore, susceptible to nutrient stress. Notably, nutrient deprivation has been correlated with poor patient survival [2], suggesting that instead of killing the tumor, the scarcity of nutrients can make the cancer cell stronger. This is likely because the existence of biochemical alterations that allow cancer cells to acquire the plasticity necessary to reprogram their metabolism in response to different nutrient conditions, positioning them better to compete, and thus resulting in a more aggressive tumor. In this regard, the work of Sun and co-workers show that when cancer cells are deprived of glucose or glutamine, the serine biosynthesis pathway (SSP) is activated [3] (Figure 1). These results confirm previous data in colon cancer cells demonstrating that glucose deprivation promotes cell death unless they are deficient in the atypical PKC, $\mathrm{PKC} \zeta$, and thus they can synthesize serine and glycine from glutamine through a process of "reverse glycolysis" [4]. Interestingly, Sun et al. [3] extended these observations and made the important finding that the SSP pathway is activated not only under glucose deprivation condition [4] but also when cells are deprived of glutamine. These observations begged the question, why is the SSP pathway so relevant? The first evidence of the importance of this pathway for tumorigenesis came from the studies of Locasale and co-workers who found that certain cancer cells utilize part of the glycolytic carbon for the serine biosynthetic pathway, which correlated with the amplification of phosphoglycerate dehydrogenase (PHGDH; Figure 1) [5]. Consistent with these data, it was also shown that cell proliferation was severely attenuated by downregulation of PHGDH in cells with an amplified PHGDH gene [5]. This suggests that the channeling of glycolytic products to this pathway might have a number of metabolic benefits that cannot be compensated by the import of extracellular serine. Interestingly, Possemato et al. [6] also established the 3-phosphoglycerate (3PG) $\rightarrow$ serine pathway as relevant to cancer, and suggested that the production of $\alpha$-ketoglutarate from glutamine- derived glutamate during the conversion of phospho-hydroxypyruvate to phospho-serine by PSAT1 was the relevant step for tumorigenesis (Figure 1). More recent data demonstrated that serinedriven one-carbon metabolism, in which oxidation of methylene tetrahydrofolate to 10-formyl-tetrahydrofolate is coupled to reduction of $\mathrm{NADP}^{+}$to NADPH, is a source of reducing potential with comparable importance to the oxidative pentose phosphate pathway [7]. This pathway also supports another critical component of the cellular redox system, glutathione biosynthesis, as glycine is one of the three amino acids (along with glutamate and cysteine) that compose glutathione. This could explain at least in part why the SSP cascade is so relevant and, according to the recent evidences, so heavily regulated.

In this regard, two very recent studies further support this notion. Gottlieb and co-workers have recently demonstrated that serine is a natural ligand of pyruvate kinase M2 (PKM2), and that serine binding allosterically activates PKM2 enzymatic activity [8]. This has important metabolic implications due to the critical role played by PKM2 in the regulation of the glycolytic flux (Figure 1). Furthermore, Thompson and co-workers presented compelling evidence that PKM2 exerts a regulatory contribution to the serine synthetic pathway [9]. Thus, in the absence of serine, the glycolytic flux to lactate is diminished due to the reduced activity of PKM2, which results in the accumulation of glycolytic intermediates that are diverted to the PHGDH-driven serine biosynthetic pathway [9]. This model implies that cancer cells, by express- 


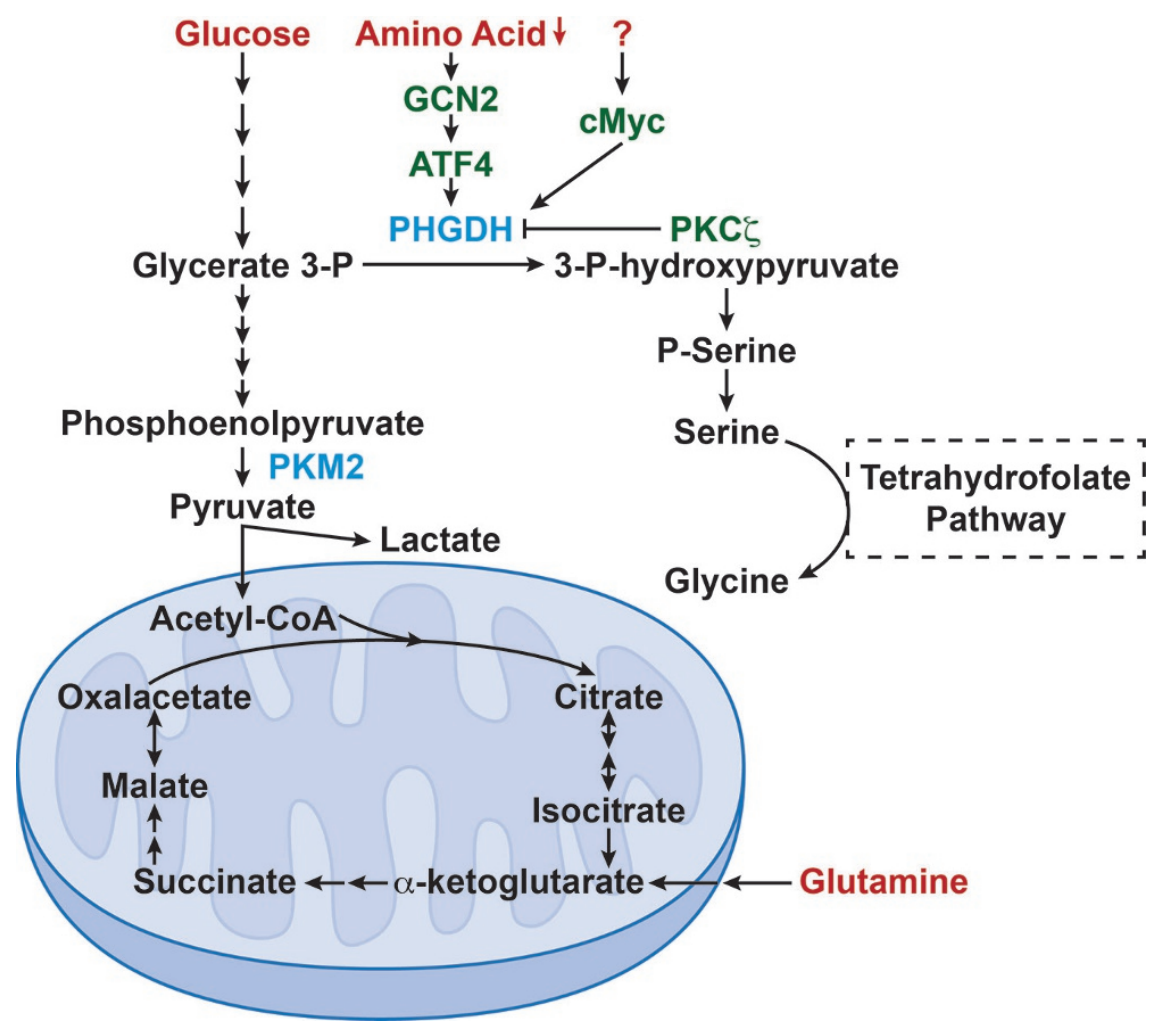

Figure 1 Nutrient sensing and stress in the serine pathway.

ing PKM2, can maintain high levels of anabolism and cell proliferation in the absence of serine in the extracellular milieu. Therefore, the crosstalk between PHGDH and PKM2 appears central to the regulation of cancer metabolism. The results of Sun et al. [3] showing that c-Myc stimulated SSP activation by transcriptionally regulating the expression of several SSP enzymes in cancer cells adds another very interesting layer of complexity to the regulation of the pathway. However, much work remains to be done to fully understand this complex regulatory cascades and lates c-Myc to impact PHGDH in the context of ATF4 activation and PKC $\zeta$ activity. Furthermore, recent data from Valencia et al. [10] demonstrated that the downregulation of c-Myc in p62/ mTORC1-deficient stromal fibroblasts results in impaired SSP that leads to more ROS and inflammation, which creates a more protumorigenic microenvironment in prostate cancer and likely in other tumors. This is important to consider because therapies aimed at blocking c-Myc or mTORC1 at a systemic level will create a more reactive tumor stroma that will likely hamper the therapeutic efficacy of these treatments in the tumor epithelium.

Jorge Moscat ${ }^{1}$, Adam Richardson ${ }^{1}$, Maria T Diaz-Meco ${ }^{1}$

${ }^{1}$ Sanford-Burnham Medical Research Institute, La Jolla, CA 92037, USA

Correspondence: Jorge Moscat

E-mail: jmoscat@sanfordburnham.org

\section{References}

1 Vander Heiden MG, Cantley LC, Thompson CB. Science 2009; 324:1029-1033.

2 Le QT, Chen E, Salim A, et al. Clin Cancer Res 2006; 12:1507-1514.

3 Sun L, Song L, Wan Q, et al. Cell Res 2015; 25:429-444.

4 Ma L, Tao Y, Duran A, et al. Cell 2013; 152:599-611.

5 Locasale JW, Grassian AR, Melman T, et al. Nat Genet 2011; 43:869-874.

6 Possemato R, Marks KM, Shaul YD, et al. Nature 2011; 476:346-350.

7 Fan J, Ye J, Kamphorst JJ, et al. Nature 2014; 510:298-302.

8 Chaneton B, Hillmann P, Zheng L, et al. Nature 2012; 491:458-462.

9 Ye J, Mancuso A, Tong X, et al. Proc Natl Acad Sci USA 2012; 109:6904-6909.

10 Valencia T, Kim JY, Abu-Baker S, et al. Cancer Cell 2014; 26:121-135. 